\title{
Epithelial IL-1R2 acts as a homeostatic regulator during remission of ulcerative colitis
}

\author{
R Mora-Buch $^{1}$, I Dotti ${ }^{1}$, N Planell ${ }^{1,2}$, E Calderón-Gómez ${ }^{1}, \mathrm{P}$ Jung $^{3}$, MC Masamunt $^{1}$, J Llach $^{4}$, E Ricart $^{1}$, \\ E Batlle $^{3,5}$, J Panés ${ }^{1}$ and A Salas ${ }^{1}$
}

Ulcerative colitis (UC) is a chronic intestinal inflammatory disease that may undergo periods of activity followed by remission. We aimed to identify the endogenous regulatory mechanisms that may promote disease remission.

Transcriptional and protein analysis of the intestinal mucosa revealed that the IL-1 decoy receptor, interleukin-1 receptor type 2 (IL1R2), was upregulated in remission compared with active UC and controls. We identified epithelial cells as being responsible for increased IL-1R2 production during remission. Expression of IL1R2 was negatively regulated by Wnt/beta-catenin signals in colonic crypts or epithelial stem cell cultures; accordingly, epithelial stem cells upregulated IL-1R2 upon differentiation. Blocking IL-1R2 in isolated colonic crypt cultures of UC patients in remission and T-cell cultures stimulated with biopsy supernatant from UC patients in remission boosted IL-1及-dependent production of inflammation-related cytokines. Finally, IL1R2 transcription was significantly lower in patients that relapsed during a 1-year follow-up period compared with those in endoscopic remission. Collectively, our results reveal that the IL-1/IL-1R2 axis is differentially regulated in the remitting intestinal mucosa of UC patients. We hypothesize that IL-1R2 in the presence of low concentrations of IL-1ß may act locally as a regulator of intestinal homeostasis.

\section{INTRODUCTION}

Ulcerative colitis (UC) is an idiopathic chronic inflammatory disease of the large intestine. In most patients, UC runs a remitting and relapsing course, with periods of active disease followed by phases of inactivity. Although current therapeutic options can induce remission in about $30-70 \%$ of patients, continuous pharmacological treatment is often required to avoid disease relapse. ${ }^{1}$ In order to promote sustained remission, endogenous mechanisms that support intestinal homeostasis and contain arising local inflammation must be identified.

In a previous study, we had shown that despite complete healing of mucosal lesions, about half of those genes de-regulated during colonic inflammation remain altered in the involved remitting mucosa of UC patients. ${ }^{2}$ Here, we further exploited the transcriptional signature of UC remission and determined that the interleukin-1 receptor type 2 gene (IL1R2), a decoy receptor for the pleiotropic cytokine IL-1 (IL- $1 \alpha$ and IL-1 $\beta$ ), was significantly upregulated during remission of the disease.
The IL-1 system comprises an array of molecules including receptors, co-receptors, ligands, and antagonists. ${ }^{3,4}$ Both the $I L 1 A$ and $I L 1 B$ genes are highly upregulated in the inflamed colonic mucosa of $\mathrm{UC}^{2,5}$ and Crohn's disease (CD). ${ }^{6} \mathrm{IL}-1$ proteins exert their effects by binding to ubiquitously expressed IL-1 receptor type 1 (IL-1R1), which associates with the IL-1R accessory protein (IL-1RAcP). In this way, it not only has crucial roles in host defense and tissue homeostasis, ${ }^{7}$ but also underlies the pathology of several auto-inflammatory conditions. ${ }^{3}$ IL-1 receptor antagonist (IL-1Ra) and IL-1R2 are negative regulators of the IL-1 cascade. IL-1R2 serves as a potent inhibitor of IL-1 signaling by competing with IL-1R1 for IL-1, and by subsequently forming a complex with IL-1RAcP, thereby sequestering both the ligand and the accessory protein required for signal transduction. ${ }^{4,8} \mathrm{IL}-1 \mathrm{R} 2$ can be expressed as a surface molecule lacking the cytoplasmic-signaling Toll/IL-1R (TIR) domain, or as a soluble IL-1 binding protein. In contrast, IL-1Ra acts predominantly as a soluble competitive inhibitor that prevents IL-1 from interacting with IL-1R1. ${ }^{3}$ Differences in

${ }^{1}$ Department of Gastroenterology, IDIBAPS, Hospital Clínic, CIBERehd, Barcelona, Spain. ${ }^{2}$ Bioinformatics Platform, CIBERehd, Barcelona, Spain. ${ }^{3}$ Oncology Program, Institute for Research in Biomedicine Barcelona (IRB Barcelona), Barcelona, Spain. ${ }^{4}$ Endoscopy Unit, Hospital Clínic, CIBERehd, Barcelona, Spain and ${ }^{5}$ Institució Catalana de Recerca i Estudis Avançats (ICREA), Barcelona, Spain. Correspondence: A Salas (asalas1@clinic.ub.es) 
their expression kinetics in response to diverse stimuli suggest that these two negative regulators of the IL-1 pathway may exert different biologic roles. Moreover, the roles of IL- $1 \alpha$ and IL- $1 \beta$ in intestinal homeostasis are still not well defined and may vary depending on their concentration and target cell(s). ${ }^{9}$

Studies have established the potent IL-1-blocking effects of IL-1Ra. ${ }^{10}$ In contrast, the only in vivo evidence available for the decoy receptor supports its regulatory effect in controlling local inflammation. ${ }^{11,12}$

Here, we provide evidence that in contrast to IL-1Ra, IL-1R2 is upregulated only during UC remission, supporting the view that these two negative regulators of IL- 1 have biologically different roles in vivo. Given that IL-1 $\beta$ is significantly downmodulated during disease remission, compared with active disease, we hypothesize that IL-1R2 represents an endogenous locally acting molecule that may counterbalance low persistent or locally arising IL- $1 \beta$ production in chronic UC patients. In order to test this hypothesis, we set out to investigate the cellular sources of IL-1R2, the pathway regulating its expression, and its functional significance during remission of UC.

\section{RESULTS}

\section{IL1R2, an IL-1 decoy receptor, is upregulated in the} intestinal mucosa of UC patients in remission

In a previous study, we showed that the involved colonic mucosa of UC patients in remission displays a characteristic transcriptional profile that differs from the mucosa of non-inflammatory bowel disease (IBD) controls or patients with active UC. ${ }^{2}$ Further analysis of these data (http://www. ncbi.nlm.nih.gov/geo/query/acc.cgi?acc=GSE38713) revealed a significant upregulation of IL1R2 (> fivefold change) in UC patients in remission compared with those with active disease, and over twofold overexpression compared with non-IBD controls or with the mucosa of uninvolved segments in patients with UC (Supplementary Figure S1 online). In contrast, $I L 1 B$, ILIA, IL1RAP, and IL1R1 genes were upregulated in the inflamed mucosa compared with all the other conditions. Transcription of the IL1R antagonist, IL1RN, was significantly upregulated in all involved mucosa independently of the presence of inflammation (Supplementary Figure S1).

In order to validate these findings, we performed RT-PCR in an independent cohort of UC patients and controls (Patient group 1, Supplementary Table S1). No significant differences in age, gender, and treatment received were found between UC groups. Patients in remission, however, had a significantly longer disease duration compared with those with active UC (Patient group 1, Supplementary Table S1). Nonetheless, we did not observe correlation between the expression patterns of any of the genes studied and disease duration. As shown in Figure 1, we confirmed that the highest expression of $I L 1 B$ was observed in the mucosa with active UC, although the expression of $I L 1 B$ in UC remission was also higher relative to controls and uninvolved UC mucosa (Figure 1a), while IL1R2 was only overexpressed in UC in remission (Figure 1b). IL1R1, a positive mediator of the IL-1 signaling cascade, was significantly upregulated in active UC compared with remission, uninvolved mucosa, and non-IBD controls (Figure 1c). IL1R1 transcription showed a moderate negative correlation with IL1R2 expression (rho $=-0.43, P=0.015$; Supplementary Figure S2). Compared with controls, IL1RN was upregulated in the involved UC mucosa, both in the presence and absence of active inflammation, thus confirming our microarray data (Figure 1e, Supplementary Figure S1). Interestingly, IL1RN transcription correlated with IL1R2 $($ rho $=0.53, P<0.01$; Supplementary Figure S2). Moreover, we identified a strong correlation between the expression of ILIRAP (Figure 1d) and ILIR1 $(r=0.68 ; P<0.001)$, both of which are required for positive IL-1 signaling (Supplementary Figure S2).

Given that $\mathrm{CD}$ is also a chronic inflammatory disease of the intestine, we tested whether these same alterations were present in the CD (Supplementary Table S2) colonic mucosa. Despite showing increased $I L 1 B$ transcription in the inflamed mucosa, CD patients did not upregulate the expression of IL1R2 during remission (Supplementary Figure S3), suggesting that the changes observed here applied exclusively to the remitting UC mucosa.

\section{Soluble IL-1R2 secretion is elevated in the involved mucosa of UC patients in remission}

Next, we measured protein secretion of soluble IL-1R2 and other IL-1 family proteins. Serum levels of IL-1 $\beta$ and soluble IL-1R2 did not relate with disease activity in UC or CD patients (Patient group 2, Supplementary Table S1, Supplementary Table S2, and Supplementary Figure S4).

Consistent with the identified transcriptional profile in UC, secretion of IL-1 $\beta$ and IL-1Ra was significantly higher in cultured biopsies from the involved colonic mucosa of patients with active disease compared with all the other conditions (Patient group 3, Supplementary Table S1; Figure 2a, d). Moreover, we detected an increase, although not significant, in soluble IL-1RAcP in inflamed UC samples (Figure 2c). Remarkably, soluble IL-1R2 was found at significantly higher concentrations in supernatants of cultured mucosal samples from UC in remission compared with active disease and uninvolved mucosa (Figure 2b). Soluble IL-1R1, however, was not detected in the biopsy culture supernatants of any patient group (data not shown), suggesting that its expression may be limited to the membrane-bound form in colonic mucosa.

Thus, while we noted a significant increase in the transcription of both IL-1 blocking genes (IL1RN and IL1R2) during remission, we only detected an increase in soluble IL-1R2 secretion. In contrast, high IL-1 $\beta$ concentrations were accompanied by increased IL-1Ra secretion in active UC.

\section{Lamina propria plasma cells and intestinal epithelial cells express IL-1R2}

To identify the cellular source of IL-1R2 in the intestinal mucosa, we carried out immunofluorescence analysis of colonic tissues. IL-1R2 ${ }^{+}$cells could be visualized both within the lamina propria, as well as in the adjacent mucosal Ep-CAM ${ }^{+}$ epithelium in non-IBD controls, UC, and CD patients (Figure 3 
a

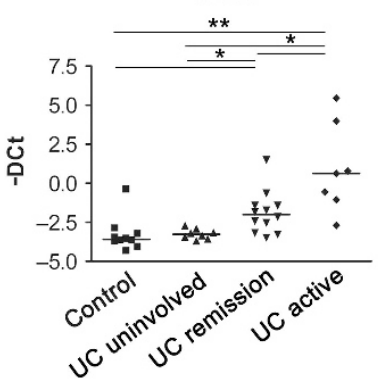

d

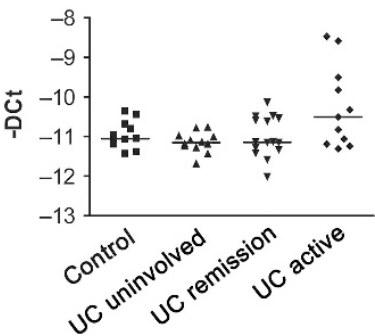

ILIRAP b

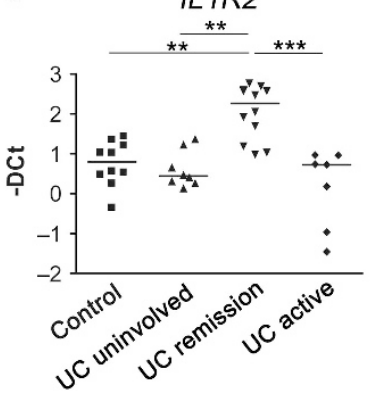

e

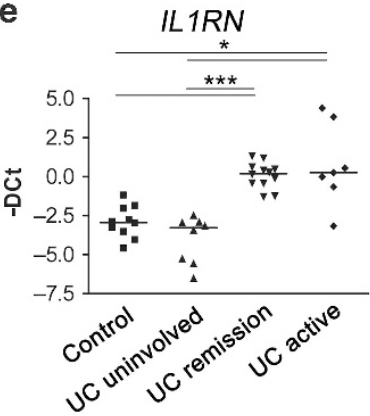

c

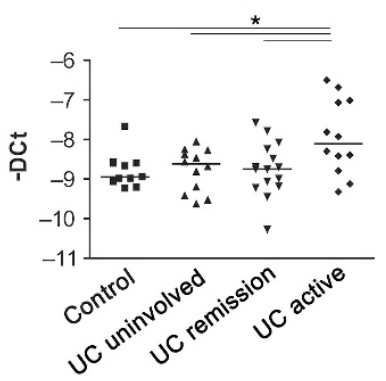

Expression of selected IL-1 family genes in colonic mucosa. (a-e) Dot plot representation (line as median) of mRNA expression of IL 1B, IL1R2, IL1R1, IL1RAP, and IL1RN as determined by GPCR (-Delta Ct) in controls $(n=10)$, uninvolved areas from patients with UC ( $n=8)$, UC patients in remission $(n=12)$, and patients with active UC $(n=7)$. Gene expression data analyzed by Kruskal-Wallis test, followed by Benjamini-Hochberg post hoc correction test. ${ }^{*} P<0.05,{ }^{* *} P<0.005,{ }^{* * *} P<0.0005$. IL, interleukin; qPCR, quantitative real-time RT-PCR; UC, ulcerative colitis. and Supplementary Figure S5b). Surprisingly, all IL-1R2 ${ }^{+}$ cells within the lamina propria showed negative (or weak) CD45 staining in healthy mucosa (Supplementary Figure S5a).

It has been described that terminally differentiated plasma cells downregulate expression of the hematopoietic marker CD45. ${ }^{13}$ Indeed, all IL-1R2 ${ }^{+}$cells within the lamina propria of healthy, UC (Figure 3), and CD patients (Supplementary Figure S5b) were identified as immunoglobulin $\mathrm{A}(\operatorname{IgA})^{+}$. No co-localization of IL-1R2 and immunoglobulin G (IgG) was observed (data not shown).

\section{Epithelial cells in UC patients in remission express increased intracellular IL-1R2}

To identify which of the two cellular subsets, plasma cells or epithelial cells, contributes to increased IL-1R2 expression in UC remission, we first quantified the number of IL-1R2 ${ }^{+}$cells within the $\operatorname{IgA}^{+}$lamina propria population in control and UC remission samples. Owing to massive cell infiltration and tissue destruction in the inflamed mucosa of UC active patients, we did not attempt to quantify the number of positively stained cells in these patients. While the total number of $\operatorname{IgA}^{+}$cells was significantly higher in the lamina propria of non-IBD control mucosa compared with UC in remission, about $50 \%$ of the $\mathrm{IgA}^{+}$population was IL-1R2 ${ }^{+}$both in controls and in UC samples (Patient group 4, Supplementary Table S1; Supplementary Figure S6).

In order to determine IL-1R2 expression by epithelial cells, we used flow cytometry to quantify IL-1R2 production by the epithelial compartment $\left(\mathrm{CD} 45^{-} \mathrm{Ep}^{-\mathrm{CAM}^{+}}\right)$in colonic samples (Patient group 5, Supplementary Table S1; Figure 4a, b). a

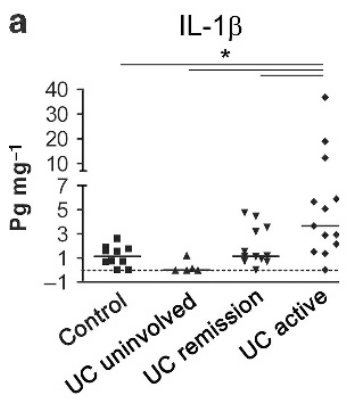

b

b Soluble IL-1R2

c

Soluble IL-1RAcP
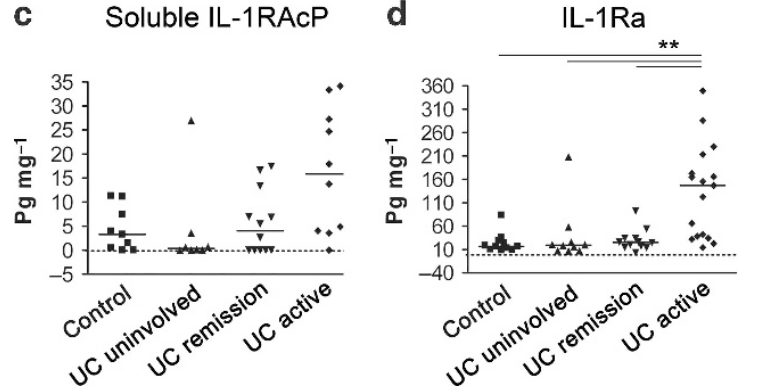

Figure 2 Secretion of selected IL-1 family proteins in colonic mucosa. (a-d) Dot plot representation (line as median) of the secretion of IL-1 $\beta$, soluble IL-1R2, soluble IL-1RAcP, and IL-1Ra in 24-h culture media of colonic biopsies from controls $(n=10)$, uninvolved areas from patients with UC $(n=11)$, UC patients with disease in remission $(n=13)$, and active UC disease mucosa $(n=17)$. Data are analyzed using a KruskalWallis test, followed by a Benjamini-Hochberg post hoc correction test. ${ }^{\star} P<0.05,{ }^{*} P<0.005$. IL, interleukin; UC, ulcerative colitis.

Samples from the involved mucosa of UC in remission showed a significantly higher percentage of intracellular, IL-1R2 ${ }^{+}$cells among $\mathrm{CD}^{-} 5^{-} \mathrm{Ep}^{-\mathrm{CAM}^{+}}$epithelial cells compared with 

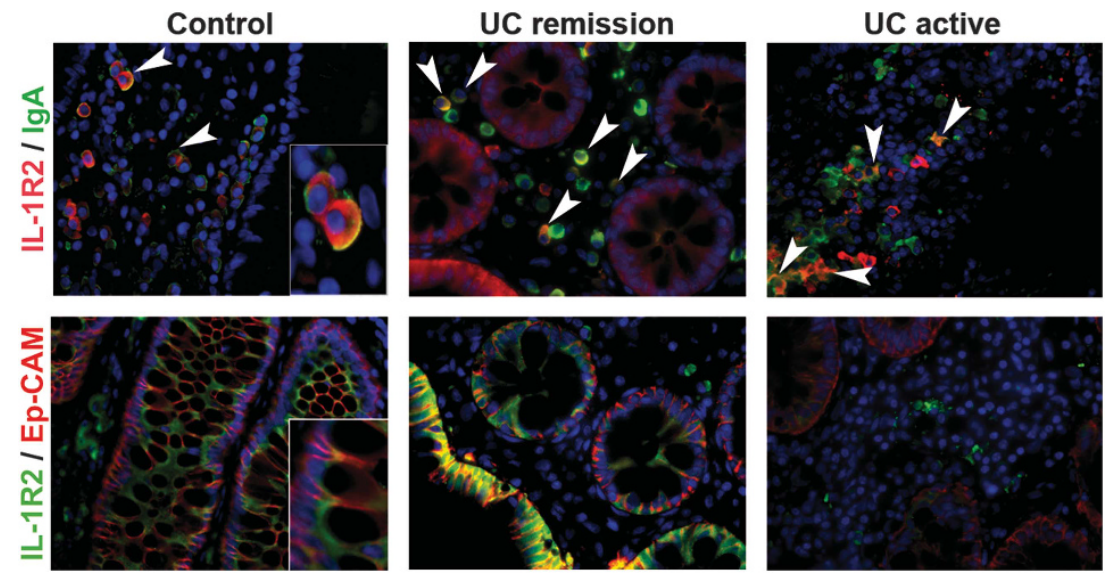

Figure 3 Immunofluorescent staining of intestinal samples shows lamina propria plasma cells and epithelial cells express IL-1R2. Representative twocolor immunofluorescent staining of fixed paraffin-embedded healthy colonic tissue, mucosa from UC patients in remission, and UC patients with active disease. The upper-right images show samples co-stained with IL-1R2 (red) and IgA (green). White arrows show IL-1R2 expression by IgA-positive cells. The bottom side images show samples co-stained with IL-1R2 (green) and Ep-CAM (red). Sections were counterstained with DAPI (blue). Images were taken with $\times 40$ objective lens. DAPI, 4',6-diamidino-2-phenylindole; Ig, immunoglobulin; IL, interleukin; UC, ulcerative colitis.

a
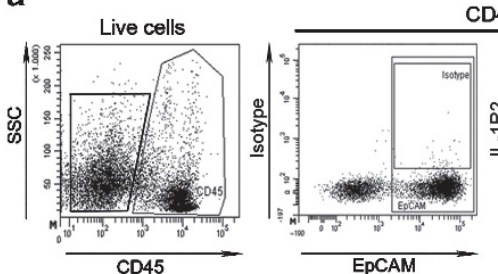

CD45-

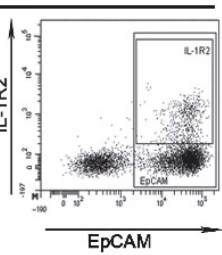

b

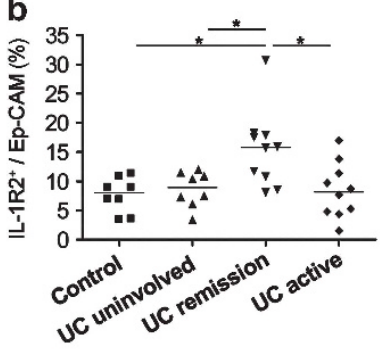

C

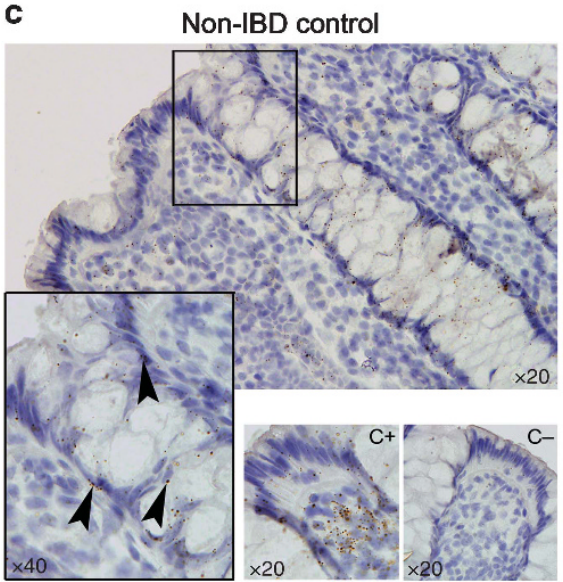

UC remission

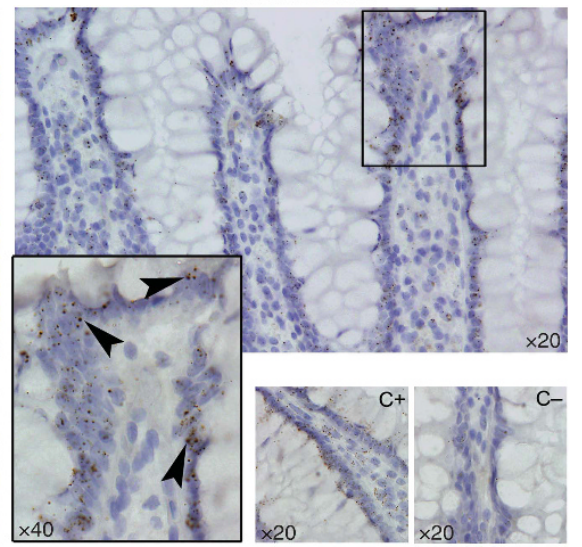

Figure 4 Increased numbers of epithelial cells express IL-1R2 in UC patients in remission. (a) Representative flow cytometry dot plots from digested biopsies. (b) Dot plot representation (line as median) of the percentages of intracellular IL-1R2 staining among Ep-CAM ${ }^{+}$from CD45 ${ }^{-}$cells from controls $(n=8)$, uninvolved mucosa from UC patients $(n=8)$, mucosa from UC patients in remission $(n=10)$, and active UC patients $(n=10)$. Data were analyzed using a Kruskal-Wallis test, followed by a Benjamini-Hochberg post-hoc correction test. ${ }^{*} P<0.05$. (c) In situ hybridization of $I L 1 R 2$ transcripts in colonic lamina propria from control and UC patient in remission. Sections were counterstained with hematoxylin. Images were taken with $\times 20$ and $\times 40$ objective lenses. Hs-PPIB probe as a positive control $(\mathrm{C}+)$ and DapB probe as a negative control $(\mathrm{C}-)$. IL, interleukin; UC, ulcerative colitis.

control, uninvolved UC, and active UC samples (Figure $4 \mathbf{b}$ ). No surface expression of the IL-1R2 was detected in epithelial cells (data not shown). To rule out the possibility that the intracellular IL-1R2 staining detected in epithelial cells was a transcytosed protein, we performed in situ hybridization. In situ staining for IL1R2 transcripts in colonic lamina propria sections confirmed ongoing ILIR 2 transcription by epithelial cells (Figure 4c). On the basis of these findings, we propose that increased expression 


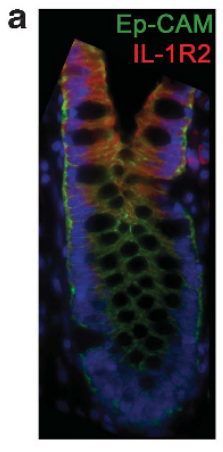

b
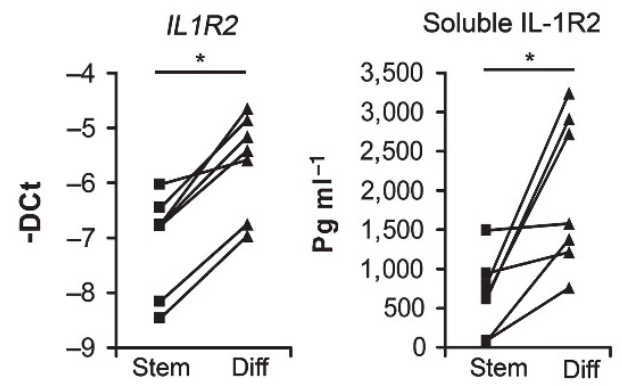

d
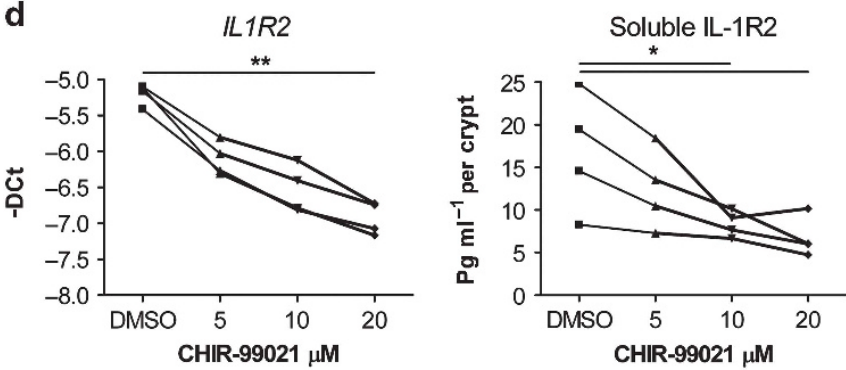

c
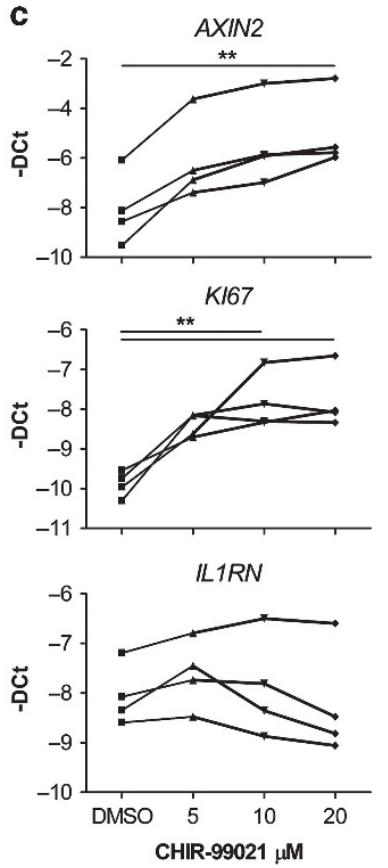

Figure 5 IL-1R2 is overexpressed by differentiated epithelial cells. (a) Representative two-color immunofluorescent staining of fixed paraffinembedded mucosa from UC remission colonic tissue. Samples were co-stained with IL-1R2 (red) and Ep-CAM (green). Section was counterstained with DAPI (blue). Image was taken with a $\times 40$ objective lens. (b) IL1R2 gene expression by qPCR ( - Delta Ct) and ELISA measurement of soluble IL-1R2 from cultured stem and differentiated (Diff) epithelial cells $(n=7)$. IL $1 R 2$ expression data were analyzed using a Wilcoxon matched pairs test. ${ }^{*} P<0.05$. (c) KI67, AXIN2, and IL1RNgene expression by qPCR ( - Delta Ct) of intestinal crypts stimulated with CHIR-99021 ( $n=4)$. (d) IL1R2 gene expression by qPCR ( - Delta Ct) and ELISA measurement of IL-1R2 secretion from intestinal crypts stimulated overnight with CHIR-99021. Gene expression data were analyzed by a Friedman test, followed by a Nemenyi post hoc test. ${ }^{\star} P<0.05$, ${ }^{\star \star} P<0.005$. IL, interleukin; qPCR, quantitative real-time RT-PCR.

of IL-1R2 in the involved mucosa of UC patients in remission is, at least in part, due to an enhanced production of the decoy receptor by epithelial cells.

\section{IL1R2 is upregulated upon differentiation of intestinal epithelial cells by inhibition of Wnt signaling}

Given the increase in epithelial IL-1R2 expression in remission, we asked which pathway drives its expression. Immunostaining analysis of colonic mucosa revealed a gradient expression of IL-1R2 along the crypt (Figure 5a). More intense IL-1R2 expression was seen at the upper half of the colonic crypts, where the differentiated epithelial cell compartment is localized, suggesting that the expression of this protein may be regulated during epithelial cell differentiation. In order to confirm this hypothesis, we used a primary epithelial organoid culture of colonic stem cells (CoSCs) from healthy mucosa. In vitro expanded CoSCs can be induced to differentiate by removing Wnt/beta-catenin activating signals from the culture media $^{14}$ (Supplementary Figure S7). Using this system, we demonstrate that both IL1R2 gene transcription and IL-1R2 protein secretion is significantly increased upon CoSCs in vitro differentiation (Figure $5 \mathbf{b}$ ). These results suggest that Wnt/beta-catenin signals, which are required for the survival and proliferation of the stem compartment at the bottom of the crypt, could be involved in repressing ILIR2 transcription and protein secretion. Indeed, isolated healthy whole intestinal crypts, cultured in the presence of Wnt/beta- catenin agonist (CHIR-99021), ${ }^{15}$ increased transcription of the proliferation marker KI67 and the beta-catenin target gene AXIN2 (Figure 5c), while at the same time, the transcription of $I L 1 R 2$, as well as the protein secretion of IL-1R2, was decreased in a dose-dependent manner (Figure 5d). On the other hand, expression of the IL-1R antagonist transcript ILIRN, which is not induced during differentiation, was not significantly influenced by CHIR-99021 (Figure 5c).

We demonstrate that IL-1R2 is suppressed by Wnt/betacatenin-dependent signals and therefore its expression is upregulated upon epithelial stem-cell differentiation.

\section{Epithelial IL-1R2 partially prevents chemokine production induced by IL-1 $\beta$ on intestinal crypts}

Next, we tested whether epithelial cell-released IL-1R2 can interfere with the effect of IL- $1 \beta$ on the intestinal epithelium. In the intestinal mucosa, IL-1 $\beta$ can be produced by immune and stromal cells within the lamina propria, but whether primary epithelial cells can produce it remains controversial. Given that we could detect no protein IL-1 $\beta$ production by isolated epithelial cells (data not shown), we stimulated intestinal crypts isolated from biopsies (Figure 6a) of healthy colonic mucosa or involved areas of UC patients in remission (Patient group 6, Supplementary Table S1) with low dose IL-1 $\beta$. We assessed the effect of an IL-1R2-blocking antibody on the expression of CXCL1, CXCL2, CXCL8, CCL20, TNFA, and IL6, pro-inflammatory chemokines highly upregulated in active 
a

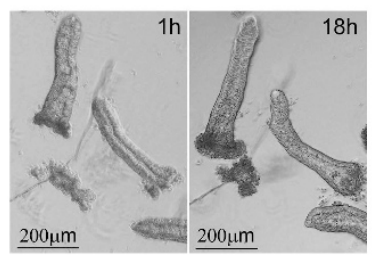

c

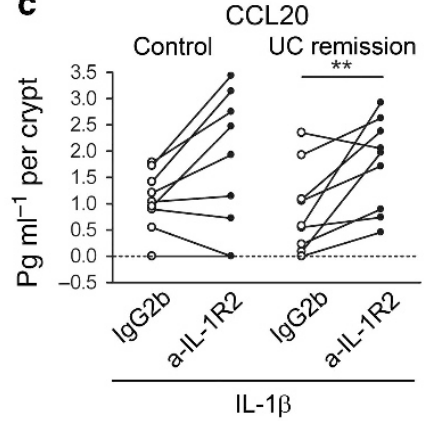

b

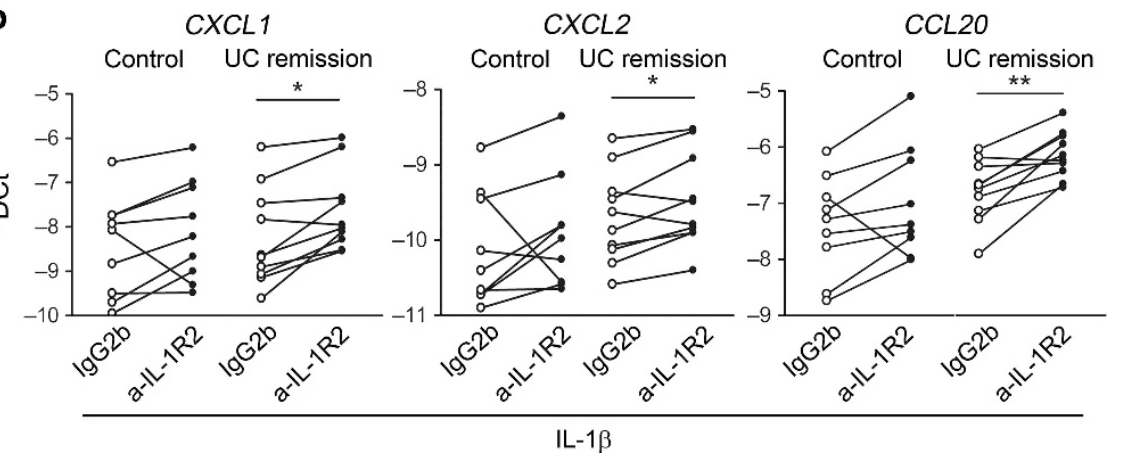

Figure 6 IL-1R2 protein partially prevents pro-inflammatory actions of IL-1 $\beta$ on intestinal crypts culture from UC patients in remission. (a) Representative image of whole intestinal crypts from colonic biopsies cultured for $18 \mathrm{~h}$. (b) CXCL1, CXCL2, and CCL20 gene expression of isolated intestinal crypts from healthy controls $(n=9)$ and UC patients in remission $(n=10)$ stimulated with IL-1 $\beta$ and IL-1R2-blocking antibody (rat anti-hIL1RII-M22) or isotype control (rat IgG2b). Determined by qPCR (-Delta Ct). (c) CCL20 secretion determined by ELISA in colon-isolated crypts from controls $(n=9)$ and UC patients in remission $(n=10)$ stimulated with IL-1 $\beta$ and with IL-1R2-blocking antibody (rat anti-hIL1RII-M22) or isotype control (rat IgG2b). Data were analyzed using a Wilcoxon matched paired test. ${ }^{\star} P<0.05,{ }^{* *} P<0.005,{ }^{* \star *} P<0.005$. IL, interleukin; qPCR, quantitative real-time RT-PCR; UC, ulcerative colitis.

UC. ${ }^{16-18}$ Transcripts for IL6 and TFNA were not detectable in isolated crypts whether resting or activated with IL-1 $\beta$. In contrast, IL-1 $\beta$ induced an increase in the transcription of CXCL1, CXCL2, CCL20, and CXCL8 in colonic crypts (data not shown). Importantly, the addition of an anti-IL-1R2-blocking antibody significantly enhanced IL- $1 \beta$-induced transcription of CXCL1, CXCL2, and CCL20 in samples from UC patients in remission (Figure 6b). CCL20 protein secretion upon IL-1 $\beta$ stimulation by intestinal crypts from UC patients in remission was also significantly increased by blocking IL-1R2 (Figure 6c). Although a similar pattern was observed in intestinal crypts from controls, the differences did not reach statistical significance.

\section{IL-1R2 produced by UC-in-remission mucosa prevents IFN- $\gamma$ production by activated $\mathrm{T}$ cells}

Next, we tested whether IL-1R2 produced in the colonic mucosa could act on neighboring immune cells. Th17/Th1 cells have been identified in the mucosa of active UC patients. ${ }^{19}$ Recent evidence suggests that IL- $1 \beta$ induces human IL-1R1 ${ }^{+}$ Th17 cells to upregulate IFN- $\gamma$ production, thereby giving rise to Th17/Th1 cells with a potentially pathogenic profile. ${ }^{20}$ In order to generate cells with that phenotype, we stimulated $\mathrm{CD}^{+}{ }^{+}$lymphocytes with Candida albicans. After expansion with yeast, about $30-40 \%$ of $\mathrm{CD}^{+}{ }^{+}$cells produced IFN- $\gamma$ (Figure 7a). Expanded T cells were stimulated with biopsy supernatant from controls and UC patients in remission (Patient group 8, Supplementary Table S1). The effect of an anti-IL-1R2-blocking antibody on IFN- $\gamma$ secretion was then
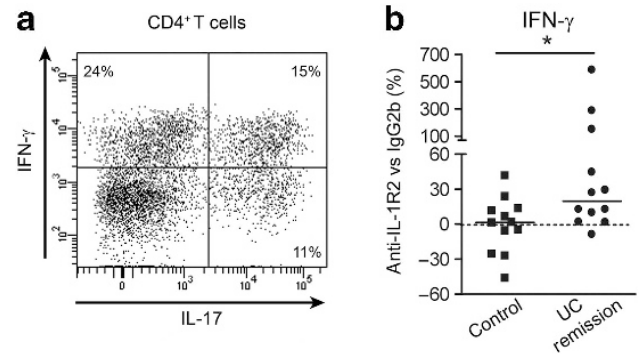

Figure 7 IL-1R2 produced by UC-in-remission mucosa prevents IFN- $\gamma$ production by $\mathrm{C}$. albicans-expanded $\mathrm{CD}^{+}{ }^{+} \mathrm{T}$ cells. (a) Representative flow cytometry dot plot from $\mathrm{CD} 4^{+}$cells expanded with $C$. albicans and autologous $\mathrm{CD} 14^{+}$monocytes. Intracellular staining of IFN- $\gamma$ and IL-17 is shown (b) C. albicans-expanded $\mathrm{CD} 4^{+} \mathrm{T}$ cells were cultured with supernatants from colonic biopsies (control; $n=12$ or UC remission; $n=12$ ) with IL-1R2-blocking antibody (rat anti-hIL1RII-M22) or the corresponding isotype control (rat IgG2b). IFN- $\gamma$ secretion was measured in culture supernatants by ELISA. Protein expression is normalized relative (\%) to the isotype control condition set at 0 (line as median). Data were analyzed using a Mann-Whitney test. ${ }^{*} P<0.05$. IFN, interferon; IL, interleukin; UC, ulcerative colitis.

determined. Blocking IL-1R2 in supernatants from UC patients in remission induced an increase in the secretion of IFN- $\gamma$ that was significantly different to the effect observed when using supernatants from control biopsies (Figure $7 \mathbf{b}$ ).

This suggests that IL-1R2 endogenously produced by mucosa from UC patients in remission acts by partially inhibiting the effects of IL-1 $\beta$ on activated T cells. 

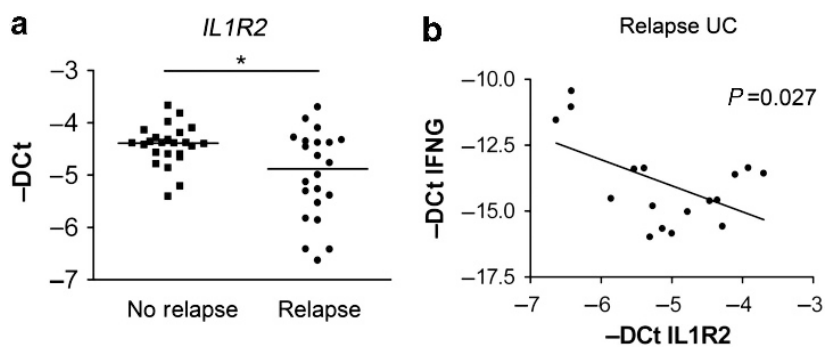

Figure 8 Lower IL1R2 gene expression in UC patients who relapsed during the following year. (a) Intestinal mucosa IL1R2 gene expression by qPCR (-Delta Ct) from UC patients in remission (line as median). Patients were classified as Relapse $(n=21)$ or No Relapse $(n=24)$ patients according to whether or not they had a disease flare (confirmed by the presence of endoscopic lesions) at some point during their 1-year followup. Gene expression data were analyzed by a Mann-Whitney test. ${ }^{*} P<0.05$. (b) IFNG and IL1R2 expression correlation in UC patients in remission that relapsed within the following year. Spearman correlation was performed ${ }^{*} P<0.05$ (Pearson $r-0.5336$ ). IL, interleukin; QPCR, quantitative real-time RT-PCR; UC, ulcerative colitis.

\section{Decreased IL1R2 gene expression is associated with relapse of UC}

Our final aim was to address whether IL-1R2 overexpression could be related to disease outcome. In order to test this possibility, we looked at IL1R2 transcription in a cohort of UC patients $(n=45)$ in endoscopic and histologic remission that were followed up for 1 year after taking biopsies from the distal colon (Patient group 7, Supplementary Table S1). Patients were classified into two groups, on the basis of whether or not they relapsed during the 12-month follow-up. Relapse was confirmed by endoscopic and histological evaluations in patients presenting symptoms, and remission was assessed by endoscopy and histology at the end of follow-up. At the time of inclusion, no significant differences existed between the two groups of patients regardless of age, gender, treatment, duration of disease, or endoscopic Mayo subscore. In addition, expression of CXCL8, CCL20, CXCL1, and CXCL2 were low and comparable between the two groups (data not shown), supporting the complete remission and lack of ongoing subclinical inflammation at the time of inclusion. Remarkably, IL1R2 transcription was significantly lower in the group of patients who relapsed during the follow-up period of 12 months compared with those patients who remained in endoscopic remission for the same amount of time (Figure 8a). Although the predictive value was low (area under the curve 0.673; 95\% confidence interval: $0.505-0.84$ ), these data suggest that IL-1R2 may have a role in preventing disease relapse. On the basis of T-cell culture results, we measured IFNG transcription in this group of patients. IFNG expression was also low and similar in two groups of inactive patients (data not shown). Interestingly, however, in patients who relapsed, IL1R2 expression negatively correlated with IFNG transcription (Figure 8b). Moreover, patients who relapsed and presented higher IL1R2 expression showed significantly lower CXCL8 expression $(P=0.0464)$ (data not shown). Altogether, our results suggest that the IL-1R2 transcriptional profile in patients with inactive disease could be an early predictor of relapse.

\section{DISCUSSION}

During acute intestinal inflammation, a number of regulatory mechanisms, such as anti-inflammatory cytokine production (IL-10, IFN- $\alpha$ and IFN- $\beta$, TGF- $\beta$, IL-22, IL-35, and IL-37), ${ }^{21}$ endogenous inhibitors of inflammation (IL-1Ra, ${ }^{2}$ sTNFR, $^{22}$ IL-18BP ${ }^{23}$ ) and pro-resolution mediators (lipoxins, resolvins, and protectins) ${ }^{24}$ are triggered, presumably to limit the inflammatory response and to regain intestinal homeostasis. Some of these mechanisms have already been explored because of their therapeutic value. ${ }^{24-26}$

The aim of our study was to reveal potential endogenous homeostatic or anti-inflammatory pathways that could be harnessed for the benefit of sustained remission. Data from several groups, including our own, ${ }^{2}$ strongly support the contention that following resolution of inflammation, a variety of signals remain deregulated in the involved colonic mucosa of UC patients. Wallace and collaborators ${ }^{27,28}$ have shown that production of the pro-resolution mediators prostaglandin D2 (PGD2), Annexin A1, and Lipoxin A4 is increased during remission of UC. Remarkably, most available data support the belief that deregulation of the intestinal epithelial compartment is maintained during disease remission. ${ }^{2,29-31}$

Here, we describe overexpression of IL-1R2 as a mechanism that is upregulated during remission in UC patients, and may block IL-1-mediated responses. IL-1 has been classically described as a pro-inflammatory cytokine promoting cell survival, cytokine and chemokine production, and increasing epithelial cell permeability. In addition, IL-1 $\beta$ participates in the differentiation of Th17 cells and has been recently described as an inducer of IFN- $\gamma$ by Th17 cells, thus giving raise to pathogenic Th1/Th17. ${ }^{20}$ Nonetheless, protective effects have also been attributed to IL-1 $\beta$ during acute colitis. ${ }^{9}$ However, data in humans that test the potential of blocking IL-1 signaling in intestinal inflammation remain lacking.

Existing data does demonstrate an overall decrease in IL-1R2 concentration during active $\mathrm{CD}$ and $\mathrm{UC}$, both in cultured colonic biopsies, ${ }^{22}$ as well as in plasma. ${ }^{32}$ Ours is the first study, however, to identify IL-1R2 as being overexpressed in colonic mucosa during disease remission, which therefore suggests that a program driving IL-1R2 production is overactive in this context. Compared with the signaling receptor IL-1R1, the decoy receptor has a 10 -fold higher affinity for IL- $1 \beta .{ }^{33}$ In addition to IL-1 binding, IL-1R2 can sequester soluble or membrane-bound IL-1RAcP, an essential component of the signaling IL-1R1 receptor. Although limited, the available in vivo data on IL-1R2 show potent local anti-inflammatory effects, ${ }^{11,12}$ suggesting that it could represent a potential therapeutic avenue.

IL-1R2 is, however, just one of multiple proteins that can affect IL-1 function. Indeed, we show that the transcription and protein expression of selected members of the IL-1 family are sequentially orchestrated in active vs. inactive disease, supporting the view that tight regulation of IL-1 signaling is crucial to maintaining and/or regaining intestinal homeostasis. The IL-1R2 gene and protein were significantly upregulated in the healed mucosa, despite concomitant downregulation of positive regulators of IL-1. This strongly suggests that IL-1R2 
may have a role in preserving intestinal homeostasis rather than in repressing ongoing acute inflammation.

To our knowledge, this is the first report to recognize the cellular sources for IL-1R2 production in the human intestine. We identified both $\mathrm{IgA}^{+}$(but not $\mathrm{IgG}^{+}$) plasma cells and mucosal epithelial cells as the main producers of IL-1R2 in human colon and provide evidence that increased IL-1R2 production during remission originates from mucosal epithelial cells. Moreover, we explore the molecular mechanism leading to IL-1R2 expression within this cellular compartment.

Previous studies have identified different signals driving IL-1R2 expression and/or shedding in isolated cells. ${ }^{34-36}$ Using an ex vivo culture of intestinal epithelial cells, we provide here novel evidence for the role of beta-catenin signaling in repressing IL-1R2 transcription and translation. Canonical Wnt signals activate beta-catenin and are critically involved in stem-cell proliferation and survival at the base of the intestinal crypts. Conversely, repression of these signals drives epithelial cell differentiation. ${ }^{15}$ Although our results do not show a correlation between different treatments and expression of $I L 1 R 2$, previous studies have demonstrated that mesalamine, commonly used to maintain UC patients in remission, inhibits epithelial beta-catenin activation in chronic UC. ${ }^{37}$ We hypothesize that increased IL-1R2 expression in the involved areas of the colon in UC in remission could reflect changes in the abundance of differentiated epithelial cells. This would be in agreement with previous data that shows permanent changes in the epithelium, ${ }^{2,29-31}$ and with the presence of aberrant crypts defined as crypt branching, the loss of parallel crypt structures, and variable crypt size, as a result of repeated crypt destruction and re-generation in UC. ${ }^{38}$ Nonetheless, further research will need to be conducted in order to fully understand the potential effects on Wnt/beta-catenin regulation in UC.

Importantly, our results demonstrate that the IL-1R2 released by the intestinal mucosa of UC patients in remission can modulate gene expression and/or protein secretion of disease-relevant mediators such as chemokines and cytokines. We propose that IL-1R2 can potentially exert a local protective role during reactivation of the disease by acting upon epithelial cells, among others, as well as upon $\mathrm{T}$ cells in the lamina propria. To prove that increased expression of IL-1R2 is functionally relevant during inactive UC remains challenging. We indirectly approached this by examining a well-characterized subset of patients ${ }^{39}$ who were in endoscopic and histologic remission at the time of endoscopic evaluation and who were followed-up with an endoscopy 12 months later. Expression of $I L 1 R 2$, albeit with a low predictive value, was significantly decreased in patients who suffered a disease flare during the subsequent 12 months. Moreover, IL1R2 expression negatively correlates with IFNG in this group of patients. This is in agreement with our hypothesis that IL-1R2 has a role in the maintenance of long-term remission, and suggests that increased IL1R 2 expression could be one, among other markers of sustained remission in UC.

Lastly, on the basis of the available data, we believe that IL-1R2 holds therapeutic potential that could be harnessed to control inflammation or to prevent relapse. IL-1-blocking strategies, such as recombinant forms of IL-1Ra (anakinra), have already been shown to have therapeutic benefits in different inflammatory conditions ${ }^{10}$ and in chronic granulomatous disease. ${ }^{40}$ IL-1R2, in contrast to IL-1Ra, offers the advantage of having a higher affinity for IL-1 $\beta$, than the signaling receptor IL-1R1, and also the ability to sequester IL-1RAcP. Administration of sIL-1R2, or of signals that promote its expression (e.g., beta-catenin antagonists already developed for colorectal cancer (CRC)), could represent an improved IL-1-blocking tool. Moreover, given the potent CRC-promoting properties of IL- $1 \beta{ }^{41}$ interfering with this cytokine could offer a dual therapeutic benefit: inhibiting/preventing inflammation, while directly interfering with cancer-promoting mechanisms. ${ }^{42,43}$ Finally, we cannot overlook the fact that IL-1 $\beta$ has been suggested to promote epithelial cell integrity in mouse models. This effect may be dependent, however, on available cytokine concentrations, concomitant expression of IL-1 regulatory molecules, and the nature of targeted cells. Further studies will be required to fully elucidate the complex behavior of IL- $1 \beta$ in mucosal homeostasis.

In summary, we propose that the secretion of IL-1R2 does not have a role in resolving or abrogating the acute inflammatory response, but rather represents a "homeostatic" mechanism that is enhanced in the previously inflamed UC mucosa. We hypothesize that boosting this mechanism could help maintain disease remission.

\section{METHODS}

More detailed information is provided in Supplementary Methods, available online only.

Patient population. Patients with an established diagnosis of UC or $\mathrm{CD}$ and non-IBD controls were included in the study after obtaining written informed consent. Non-IBD controls were those subjects undergoing surgery for CRC, colonoscopy for mild gastrointestinal symptoms, or a screening for CRC and who presented no lesions during examination. A total of 266 subjects were included in the study. Supplementary Table S1 shows the clinical and demographic characteristics from non-IBD controls and UC patients; samples were randomly distributed in eight independent subgroups on the basis of the different experimental approaches used. CD patient characteristics are included in Supplementary Table S2.

Assessment of disease activity. Endoscopic activity at the time of colonoscopy was categorized according to the Mayo endoscopic subscore. ${ }^{44}$ Active disease was defined as a Mayo endoscopic subscore of 1-2-3; quiescent disease (remission) was defined as a Mayo score of 0 or 1 with limited erythema in a segment with evidence of active disease in any previous endoscopy; a segment was categorized as uninvolved when no lesions were identified in the current and any previous endoscopy.

Intestinal sample collection. Intestinal biopsies from the sigmoid colon were collected during routine colonoscopies from non-IBD controls, UC patients with quiescent disease, and UC patients with endoscopic activity. Samples from uninvolved segments were obtained from both UC patients in remission and from those with active disease. None of the biopsies obtained from the vicinity of samples used for the experiments described below showed evidence of colitis-associated dysplasia or neoplasia. For organoid culture and Wnt/beta-catenin agonist experiments, whole intestinal crypts were isolated from the healthy mucosa of 11 CRC patients undergoing surgery. 
Measurement of soluble proteins. Serum and culture supernatants from biopsies, from intestinal crypt cultures, and T-cell cultures were harvested, centrifuged, and stored at $-20^{\circ} \mathrm{C}$ until they were assayed for soluble IL-1R2, IL-1Ra, soluble IL-1sRAcP, soluble IL-1R1, IL-1 $\beta$, CCL20, or IFN- $\gamma$ using ELISA (R\&D Systems, Minneapolis, MN; eBioscience, San Diego, CA; BD Bioscience, San Jose, CA)

RNA isolation. Biopsies were placed in RNA Stabilization Reagent (Qiagen, Hilden, Germany). Total RNA from biopsies and matrigel (BD Biosciences) embedded crypts were isolated using the Rneasy Kit (Qiagen).

Biopsy cell isolation and flow cytometry. Intestinal biopsies were washed and digested with collagenase (Sigma, San Louis, MO) and DNAse (Roche, Basel, Switzerland); cells were stained using directly labeled antibodies. Samples were acquired in a Canto II (BD Bioscience) cytometer equipped with FACS Diva 6.1.2 software (BD Bioscience).

Colonic crypt isolation and culture. Crypts were isolated from intestinal tissues as previously described. ${ }^{14}$

For short-term crypt culture, about 30 isolated crypt $/ 25 \mu$ matrigel were plated and cultured overnight in "complete crypt culture medium". Supernatants were harvested, centrifuged, and stored at $-20^{\circ} \mathrm{C}$ until there were later assayed.

Colonic epithelial stem-cell culture. In order to obtain threedimensional organoid cultures of CoSCs, about 30 purified human colon crypts per well embedded in matrigel were overlaid with $250 \mu \mathrm{l}$ "stem medium", maintained, and passaged as previously described, ${ }^{14}$ and CoSC were thereby induced to differentiate.

Quantitative real-time RT-PCR (qPCR). Total RNA was transcribed to cDNA using reverse transcriptase (High Capacity cDNA Archive RT kit, Applied Biosystems, Carlsbad, CA). PCR was performed in TaqMan Universal PCR Master Mix (Applied Biosystems) according to the manufacturer's instructions. Fluorescence was detected using an ABI PRISM 7500 Fast RT-PCR System (Applied Biosystems).

Immunostaining of intestinal samples. Immunofluorescence staining was performed using anti-IL-1R2 (Sigma) and anti-CD45 (BD Biosciences), anti-IgA (Southern Biotech, Birmingham, AL) or anti-Ep-CAM (Dako, Carpinteria, CA). Image acquisition and overlay was performed on an Olympus (Tokyo, Japan) BX51 microscope using CellF Software.

RNA chromogenic in situ hybridization of intestinal samples. The RNAscope 2.0 assay was performed according to the supplier's instructions (Advanced Cell Diagnostics, Hayward, CA) using a custom-designed probe to detect IL-1R2 mRNA (Advanced Cell Diagnostics).

T-cell culture. Co-cultures of CD ${ }^{+}{ }^{+} \mathrm{T}$ cells and $\mathrm{CD} 14^{+}$monocytes isolated from peripheral blood mononuclear cells obtained from a buffy coat were stimulated with Candida albicans to obtain Th1, Th17, and Th1/17 cell populations. Once expanded, T cells were cultured with biopsy supernatants in the presence of IL-1R2-blocking antibody (rat anti-hIL1RII-M22; kindly provided by AMGEN, Seattle, WA) or the isotype control (rat IgG2b, eBioscience).

Statistical analysis. Numeric data are described as median and range, and categorical variables as absolute frequencies. Mann-Whitney test was performed to examine statistically different expression patterns between two groups, and a Kruskal-Wallis test was performed to examine statistical significance in multiple group data sets, followed by a Benjamini-Hochberg post hoc correction test. A Friedman test was performed to examine statistical significance in repeated-measures analysis, followed by the Nemenyi post hoc test. A Wilcoxon matchedpair test was performed to examine statistical significance in paired data. A $P$-value of $<0.05$ was considered statistically significant. Spearman correlation was performed for numeric variables. The receiver-operator characteristic area under the curve was calculated to assess the usefulness of IL1R2 expression for predicting relapse. Data were analyzed using R (version 3.1.0) (Published Online First: 2014. http://www.r-project.org/).

Ethical considerations. This study was approved by the Institutional Ethics Committee of the Hospital Clinic of Barcelona (Spain) on March 2006 and was performed in accordance with the principles stated in the Declaration of Helsinki (updated October 1996). All patients signed an informed consent prior to their inclusion in the study.

SUPPLEMENTARY MATERIAL is linked to the online version of the paper at http://www.nature.com/mi

\section{ACKNOWLEDGMENTS}

This work was supported by grants BFU2012-35999/BFI, MC1_TV3_122931, and the Helmsley Trust. R.M.B. is a recipient of a FPI fellowship from the Ministerio de Economía y Competitividad (BES2010-030033). We thank the Biobank and Citomics Facilities at the Institut d'Investigacions Biomèdiques August $\mathrm{Pi}$ i Sunyer (IDIBAPS) for their support, the Endoscopy Department at Hospital Clinic Barcelona for providing us with the samples required to conduct this study, and our patients for their selfless participation. We are indebted to Joe Moore for editorial assistance.

\section{DISCLOSURE}

The authors declared no conflict of interest.

c) 2016 Society for Mucosal Immunology

\section{REFERENCES}

1. Ordas, I., Eckmann, L., Talamini, M., Baumgart, D.C. \& Sandborn, W.J. Ulcerative colitis. Lancet 380, 1606-1619 (2012).

2. Planell, N. et al. Transcriptional analysis of the intestinal mucosa of patients with ulcerative colitis in remission reveals lasting epithelial cell alterations. Gut 62, 967-976 (2013).

3. Dinarello, C.A. Immunological and inflammatory functions of the interleukin-1 family. Annu. Rev. Immunol. 27, 519-550 (2009).

4. Garlanda, C., Dinarello, C.A. \& Mantovani, A. The interleukin-1 family: back to the future. Immunity 39, 1003-1018 (2013).

5. Roman, J. et al. Evaluation of responsive gene expression as a sensitive and specific biomarker in patients with ulcerative colitis. Inflamm. Bowel Dis. 19, 221-229 (2013).

6. Leal, R.F. et al. Identification of inflammatory mediators in patients with Crohn's disease unresponsive to anti-TNFalpha therapy. Gut 64, 233-242 (2014).

7. Lopetuso, L.R., Chowdhry, S. \& Pizarro, T.T. Opposing functions of classic and novel IL-1 family members in gut health and disease. Front. Immunol. 4, 181 (2013).

8. Colotta, F. et al. Interleukin-1 type II receptor: a decoy target for IL-1 that is regulated by IL-4. Science 261, 472-475 (1993).

9. Bersudsky, M. et al. Non-redundant properties of IL-1alpha and IL-1beta during acute colon inflammation in mice. Gut 63, 598-609 (2014).

10. Dinarello, C.A., Simon, A. \& van der Meer, J.W. Treating inflammation by blocking interleukin-1 in a broad spectrum of diseases. Nat. Rev. Drug Discov. 11, 633-652 (2012).

11. Rauschmayr, T., Groves, R.W. \& Kupper, T.S. Keratinocyte expression of the type 2 interleukin 1 receptor mediates local and specific inhibition of interleukin 1-mediated inflammation. Proc. Natl Acad. Sci. USA 94, 58145819 (1997).

12. Bessis, N. et al. The type II decoy receptor of IL-1 inhibits murine collageninduced arthritis. Eur. J. Immunol. 30, 867-875 (2000).

13. Caraux, A. et al. Circulating human B and plasma cells. Age-associated changes in counts and detailed characterization of circulating normal CD138- and CD138 + plasma cells. Haematologica 95, 1016-1020 (2010).

14. Jung, P. et al. Isolation and in vitro expansion of human colonic stem cells. Nat. Med. 17, 1225-1227 (2011).

15. Clevers, H. \& Nusse, R. Wnt/beta-catenin signaling and disease. Cell 149, 1192-1205 (2012). 
16. Zahn, A. et al. Transcript levels of different cytokines and chemokines correlate with clinical and endoscopic activity in ulcerative colitis. BMC Gastroenterol. 9, 13 (2009).

17. Egesten, A. et al. The proinflammatory CXC-chemokines GRO-alpha/ CXCL1 and MIG/CXCL9 are concomitantly expressed in ulcerative colitis and decrease during treatment with topical corticosteroids. Int. J. Colorectal Dis. 22, 1421-1427 (2007).

18. Kaser, A. et al. Increased expression of CCL20 in human inflammatory bowel disease. J. Clin. Immunol. 24, 74-85 (2004).

19. Globig, A.M. et al. Comprehensive intestinal T helper cell profiling reveals specific accumulation of IFN-gamma + IL-17 + coproducing CD4 + T cells in active inflammatory bowel disease. Inflamm. Bowel Dis. 20, 23212329 (2014).

20. Duhen, T. \& Campbell, D.J. IL-1beta promotes the differentiation of polyfunctional human CCR $6+$ CXCR3 + Th1/17 cells that are specific for pathogenic and commensal microbes. J. Immunol. 193, 120-129 (2014).

21. Neurath, M.F. Cytokines in inflammatory bowel disease. Nat. Rev. Immunol. 14, 329-342 (2014).

22. Gustot, T. et al. Profile of soluble cytokine receptors in Crohn's disease. Gut 54, 488-495 (2005).

23. Liang, D., Ma, W., Yao, C., Liu, H. \& Chen, X. Imbalance of interleukin 18 and interleukin 18 binding protein in patients with lupus nephritis. Cell. Mol. Immunol. 3, 303-306 (2006).

24. Serhan, C.N. Pro-resolving lipid mediators are leads for resolution physiology. Nature 510, 92-101 (2014).

25. Fedorak, R.N. et al. Recombinant human interleukin 10 in the treatment of patients with mild to moderately active Crohn's disease. The Interleukin 10 Inflammatory Bowel Disease Cooperative Study Group. Gastroenterology 119, 1473-1482 (2000).

26. van de Veerdonk, F.L., Netea, M.G., Dinarello, C.A. \& van der Meer, J.W. Anakinra for the inflammatory complications of chronic granulomatous disease. Neth. J. Med. 69, 95 (2011).

27. Vong, L., Ferraz, J.G., Panaccione, R., Beck, P.L. \& Wallace, J.L. A proresolution mediator, prostaglandin $\mathrm{D}(2)$, is specifically up-regulated in individuals in long-term remission from ulcerative colitis. Proc. Natl Acad. Sci. USA 107, 12023-12027 (2010).

28. Vong, L. et al. Up-regulation of Annexin-A1 and lipoxin A(4) in individuals with ulcerative colitis may promote mucosal homeostasis. PLoS One 7, e39244 (2012).

29. Treton, X. et al. Altered endoplasmic reticulum stress affects translation in inactive colon tissue from patients with ulcerative colitis. Gastroenterology 141, 1024-1035 (2011).

30. Gustafsson, J.K., Hansson, G.C. \& Sjovall, H. Ulcerative colitis patients in remission have an altered secretory capacity in the proximal colon despite macroscopically normal mucosa. Neurogastroenterol. Motil. 24, e381e391 (2012).

31. Fasseu, M. et al. Identification of restricted subsets of mature microRNA abnormally expressed in inactive colonic mucosa of patients with inflammatory bowel disease. PLoS One, 5 (2010).

32. Ludwiczek, O. et al. Imbalance between interleukin-1 agonists and antagonists: relationship to severity of inflammatory bowel disease. Clin. Exp. Immunol. 138, 323-329 (2004).
33. McMahan, C.J. et al. A novel IL-1 receptor, cloned from B cells by mammalian expression, is expressed in many cell types. EMBO J. 10, 2821-2832 (1991).

34. Lukiw, W.J., Martinez, J., Pelaez, R.P. \& Bazan, N.G. The interleukin-1 type 2 receptor gene displays immediate early gene responsiveness in glucocorticoid-stimulated human epidermal keratinocytes. J. Biol. Chem. 274, 8630-8638 (1999).

35. Penton-Rol, G. et al. Bacterial lipopolysaccharide causes rapid shedding, followed by inhibition of mRNA expression, of the IL-1 type II receptor, with concomitant up-regulation of the type I receptor and induction of incompletely spliced transcripts. J. Immunol. 162, 2931-2938 (1999).

36. Orlando, S. et al. TNF-alpha, unlike other pro- and anti-inflammatory cytokines, induces rapid release of the IL-1 type II decoy receptor in human myelomonocytic cells. J. Immunol. 158, 3861-3868 (1997).

37. Brown, J.B. et al. Mesalamine inhibits epithelial beta-catenin activation in chronic ulcerative colitis. Gastroenterology 138, 605 e591-e593 (2010).

38. Seldenrijk, C.A., Morson, B.C., Meuwissen, S.G., Schipper, N.W., Lindeman, J. \& Meijer, C.J. Histopathological evaluation of colonic mucosal biopsy specimens in chronic inflammatory bowel disease: diagnostic implications. Gut 32, 1514-1520 (1991).

39. Jauregui-Amezaga, A. et al. Accuracy of advanced endoscopy and fecal calprotectin for prediction of relapse in ulcerative colitis: a prospective study. Inflamm. Bowel Dis. 20, 1187-1193 (2014).

40. de Luca, A. et al. IL-1 receptor blockade restores autophagy and reduces inflammation in chronic granulomatous disease in mice and in humans. Proc. Natl Acad. Sci. USA 111, 3526-3531 (2014).

41. Li, Y., Wang, L., Pappan, L., Galliher-Beckley, A. \& Shi, J. IL-1beta promotes stemness and invasiveness of colon cancer cells through Zeb1 activation. Mol. Cancer 11, 87 (2012).

42. Wang, Y. et al. Neutrophil infiltration favors colitis-associated tumorigenesis by activating the interleukin-1 (IL-1)/IL-6 axis. Mucosal Immunol. 7, 11061115 (2014).

43. Dinarello, C.A. Why not treat human cancer with interleukin-1 blockade? Cancer Metastasis Rev. 29, 317-329 (2010).

44. Schroeder, K.W., Tremaine, W.J. \& Ilstrup, D.M. Coated oral 5-aminosalicylic acid therapy for mildly to moderately active ulcerative colitis. A randomized study. N. Engl. J. Med. 317, 1625-1629 (1987).

(c)(1)(2) This work is licensed under a Creative Commons Attribution-NonCommercial-ShareAlike 4.0 International License. The images or other third party material in this article are included in the article's Creative Commons license, unlessindicated otherwise in the creditline; if the material is not included under the Creative Commons license, users will need to obtain permission from the license holder to reproduce the material. To view a copy of this license, visit http:// creativecommons.org/licenses/by-nc-sa/4.0/ 